\title{
Lamb Waves in a Functionally Graded Composite Plate with Nonintegral Power Function Volume Fractions
}

\author{
Xiaoshan Cao, ${ }^{1,2}$ Zhen Qu, ${ }^{1}$ Junping Shi, ${ }^{1}$ and Yan $R u^{1}$ \\ ${ }^{1}$ Department of Engineering Mechanics, School of Civil Engineering and Architecture, Xian University of Technology, \\ Xian 710048, China \\ ${ }^{2}$ State Key Laboratory for Strength and Vibration of Mechanical Structure, Xian Jiaotong University, Xian 710049, China
}

Correspondence should be addressed to Xiaoshan Cao; caoxsh@xaut.edu.cn

Received 11 July 2014; Accepted 26 August 2014

Academic Editor: Xing Chen

Copyright (C) 2015 Xiaoshan Cao et al. This is an open access article distributed under the Creative Commons Attribution License, which permits unrestricted use, distribution, and reproduction in any medium, provided the original work is properly cited.

\begin{abstract}
An analytical modelling is carried out to determine the Lamb wave's propagation behavior in a thermal stress relaxation type functionally graded material (FGM) plate, which is a composite of two kinds of materials. The mechanical parameters depend on the volume fractions, which are nonintegral power functions, and the gradient coefficient is the power value. Based on the theory of elastodynamics, differential equations with variable coefficients are established. We employ variable substitution for theoretical derivations to solve the ordinary differential equations with variable coefficients using the Taylor series. The numerical results reveal that the dispersion properties in some regions are changed by the graded property, the phase velocity varies in a nonlinear manner with the gradient coefficient, nondispersion frequency exists in the first mode, and the set of cutoff frequencies is a union of two series of approximate arithmetic progressions. These results provide theoretical guidance not only for the experimental measurement of material properties but also for their nondestructive testing.
\end{abstract}

\section{Introduction}

Since 1990s, functionally graded materials (FGMs) have attracted the interest of researchers from many engineering fields $[1,2]$. The most popular application of FGMs is in thermal protection systems in aerospace structures. Composed of a mixture of a kind of metal and ceramic, their compositions vary from a ceramic-rich surface to a metal-rich surface. The volume fractions of metal and ceramic are functions of thickness, including polynomial, exponential, and power series functions [3-6].

In recent years, much attention has been devoted to guided waves in FGM structures for nondestructive evaluation. Many numerical solutions have been undertaken to divide an inhomogeneous medium into a multilayer model to investigate the wave propagation in inhomogeneous media [7-12]. A homogenous assumption of the material parameters in each layer has been adopted for analysis by virtue of the finite element method. Many numerical methods, such as the finite element method, the transfer matrix method, and the scaled boundary finite element method, have been employed in the research of Lamb waves propagating in an FGM plate [7-10]. Analytical solution has also been carried out on wave propagation problems in inhomogeneous structures. Several researchers have investigated various analytical solutions, such as the Wentzel-Kramers-Brillouin (WKB) method, special functions method, perturbation technique, the Legendre orthogonal polynomial series expansion, and the power series technique [13-18]. The WKB method is consistently used in investigating horizontal shear waves in an inhomogeneous layered structure [13]. Special functions, such as the cylindrical and Airy functions, are applied to the solution of ordinary different equations when the variation in material parameters follows certain specific patterns $[14,15]$. The perturbation method is used to solve the propagation problem where only one parameter varies slightly and smoothly [16]. Wu et al. studied the wave propagation problem in a functionally graded magnetoelectroelastic plate in electric and magnetic open boundary conditions using the Legendre orthogonal polynomial series expansion [17]. In this study, the material parameters are expressed as the Legendre polynomials. Hence, the material parameters may also be 
expressed as a Taylor series expansion. The power series technique is applied to the solution of guided waves in functionally graded layered structures when each material parameter can be rewritten as a Taylor series expansion [18]. Thus, this technique is suitable for the case where volume fractions are linear functions, polynomial functions, exponential functions, and so on. Based on these methods mentioned above, the Lamb wave's propagation behavior in various inhomogeneous plates or thin films has received much attention.

However, in engineering applications, the volume fractions are always power functions and the power value is not an integral [6]. Thus far, no report has yet been published on the analytical solution of the wave propagation behavior in a functionally graded composite with nonintegral power function volume fractions. In this study, we suppose that variations in material parameters occur according to the volume fractions, which are power functions of thickness with a noninteger power. Using the power series technique and variable substitution for theoretical derivations, we investigate the propagation behavior of Lamb waves in an inhomogeneous plate, including their variation in phase velocity, dispersion properties, nondispersion frequency, and cutoff frequency.

\section{Statement of the Problem}

Lamb waves propagating in an FGM plate, as shown in Figure 1, are considered in this study. The thickness of the FGM plate is $h$ and the bottom and upper surfaces of the plate are traction-free.

The constitutive equations of the FGM plate can be expressed as

$$
\sigma_{i j}=c_{i j k l} S_{k l} \text {, }
$$

where $\sigma_{i j}$ and $S_{k l}$ are the stress and strain tensors, respectively, and $c_{i j k l}$ is the elastic coefficient, which varies continuously along the thickness direction.

The equation of motion is given by

$$
\sigma_{i j, j}=\rho \ddot{u}_{i},
$$

where $\rho$ is the mass density, $u_{i}$ is the mechanical displacement component in the $i$ th direction, a comma followed by the subscript $i$ denotes space differentiation with respect to the corresponding coordinate $x_{i}$, "the dot" represents the time differentiation, and the repeated index in the subscript implies the summation with respect to that index.

The mechanical displacement is related to the strain by

$$
S_{i j}=\frac{1}{2}\left(u_{i, j}+u_{j, i}\right) .
$$

Without loss of generality, the Lamb wave is assumed to propagate along the positive direction of the $x$-axis. The mechanical displacement components and electrical potential may then be expressed as

$$
u=u(x, z, t), \quad v=0, \quad w=w(x, z, t) .
$$

Upon the following sequence of substitutions: (i) (4) into (3), (ii) modified (3) into (1), and (iii) new equation into (2),

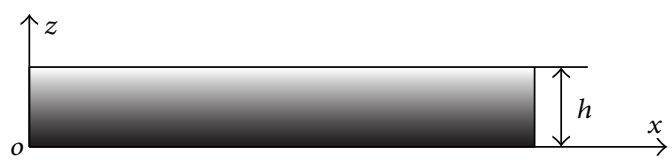

FIGURE 1: FGM plate and Cartesian coordinates.

the following governing equations for the mechanical displacements can be obtained:

$$
\begin{aligned}
& c_{11} \frac{\partial u}{\partial^{2} x}+c_{13} \frac{\partial w}{\partial x \partial z}+c_{44}\left(\frac{\partial u}{\partial^{2} z}+\frac{\partial w}{\partial x \partial z}\right)+c_{44}^{\prime}\left(\frac{\partial u}{\partial z}+\frac{\partial w}{\partial x}\right) \\
& =\rho \ddot{u} \\
& c_{44}\left(\frac{\partial u}{\partial z \partial x}+\frac{\partial w}{\partial^{2} x}\right)+c_{13} \frac{\partial u}{\partial x \partial z}+c_{33} \frac{\partial w}{\partial^{2} z}+c_{13}^{\prime} \frac{\partial u}{\partial x}+c_{33}^{\prime} \frac{\partial w}{\partial z} \\
& =\rho \ddot{w},
\end{aligned}
$$

where $c_{13}=c_{11}-2 c_{44}$.

For Lamb waves propagating in the FGM plate, the traction-free boundary conditions at the upper and bottom surfaces should be satisfied as follows:

$$
\begin{array}{ll}
\sigma_{z}(x, 0)=0, & \tau_{x z}(x, 0)=0, \\
\sigma_{z}(x, h)=0, & \tau_{x z}(x, h)=0 .
\end{array}
$$

2.1. Analytical Solutions. With reference to Figure 1, the solution of Lamb waves propagating along the positive $x$-axis of the FGM plate can be expressed as

$$
\begin{gathered}
u(x, z, t)=U(z) \exp [i k(x-c t)] \\
w(x, z, t)=i W(z) \exp [i k(x-c t)],
\end{gathered}
$$

where $i=\sqrt{-1}$ and $k=2 \pi / \lambda$ are the wave numbers, with $\lambda$ being the wavelength, $c$ is the phase velocity, and $U(z)$ and $W(z)$ are amplitudes of the unknown amplitudes of the displacement components.

Motion equations in the FGM plate can be obtained by substituting (7) into (5) as

$$
\begin{aligned}
& c_{44} U^{\prime \prime}+c_{44}^{\prime} U^{\prime}+\left(\rho c^{2}-c_{11}\right) k^{2} U-\left(c_{11}-c_{44}\right) k W^{\prime} \\
& -c_{44}^{\prime} k W=0 \\
& c_{11} W^{\prime \prime}+c_{11}^{\prime} W^{\prime}+\left(\rho c^{2}-c_{44}\right) k^{2} W+\left(c_{11}-c_{44}\right) k U^{\prime} \\
& +\left(c_{11}-2 c_{44}\right)^{\prime} k U=0 .
\end{aligned}
$$

Normally, an FGM is a functionally graded composite of two kinds of materials, materials I and II, with varying thicknesses of the volume fraction of the materials. The parameters of the FGM plate are described as

$$
g(z)=g^{(1)} f^{(1)}\left(\frac{z}{h}\right)+g^{(2)} f^{(2)}\left(\frac{z}{h}\right)
$$


where $f^{(1)}$ and $f^{(2)}$ represent the volume fractions and $g^{(1)}$ and $g^{(2)}$ indicate the parameters of materials I and II, respectively.

Some scientists have studied a kind of FGM in which volume fraction can be expressed as a polynomial. Others have investigated the FGM using volume fraction as the exponential function. In these studies, the volume fraction of materials may be expressed by Taylor series. Considering engineering applications, we suppose that the volume fraction of materials should satisfy

$$
f^{(2)}=\left(\frac{z}{h}\right)^{p}, \quad f^{(1)}=1-f^{(2)}=1-\left(\frac{z}{h}\right)^{p},
$$

where $p$ is the gradient coefficient that is expressed as $p=$ $N / M$. Therefore, the material parameters of the FGM plate are assumed to be of the following functional form:

$$
\begin{gathered}
c_{44}=\alpha_{0}+\alpha_{1}\left(\frac{z}{h}\right)^{N / M}, \quad c_{11}=\beta_{0}+\beta_{1}\left(\frac{z}{h}\right)^{N / M}, \\
\rho=\gamma_{0}+\gamma_{1}\left(\frac{z}{h}\right)^{N / M},
\end{gathered}
$$

where $\alpha_{0}, \beta_{0}$, and $\gamma_{0}$ are $c_{44}^{(1)}, c_{11}^{(1)}$, and $\rho^{(1)}$ and $\alpha_{0}+\alpha_{1}, \beta_{0}+\beta_{1}$, and $\gamma_{0}+\gamma_{1}$ are $c_{44}^{(2)}, c_{11}^{(2)}$, and $\rho^{(2)}$, respectively.

In view of the material properties given in (11), we define

$$
\widehat{z}=\left(\frac{z}{h}\right)^{1 / M}
$$

The following relation should be satisfied by

$$
\begin{gathered}
\frac{d F}{d z}=\frac{d F}{d \widehat{z}} \frac{d \widehat{z}}{d z}=\frac{1}{M h} \widehat{z}^{(1-M)} \frac{d F}{d \widehat{z}} \\
\frac{d^{2} F}{d z^{2}}=\left(\frac{1}{M h}\right)^{2}\left[(1-M) \widehat{z}^{(1-2 M)} \frac{d F}{d \widehat{z}}+\widehat{z}^{2(1-M)} \frac{d^{2} F}{d \widehat{z}^{2}}\right]
\end{gathered}
$$

where $F$ is an arbitrary derivable function of $z$.

Therefore, (8) can be rewritten as equations with respect to $\widehat{z}$ as follows:

$$
\begin{aligned}
& \left(\frac{1}{M h}\right)^{2}\left(\alpha_{0}+\alpha_{1} \widehat{z}^{N}\right)\left[\widehat{z}^{2(1-M)} \frac{d^{2} U}{d \widehat{z}^{2}}+(1-M) \widehat{z}^{(1-2 M)} \frac{d U}{d \widehat{z}}\right] \\
& +\left(\frac{1}{M h}\right)^{2} \widehat{z}^{2(1-M)} \alpha_{1} N \widehat{z}^{(N-1)} \frac{d U}{d \widehat{z}} \\
& +\left[\left(\gamma_{0}+\gamma_{1} \widehat{z}^{N}\right) c^{2}-\left(\beta_{0}+\beta_{1} \widehat{z}^{N}\right)\right] k^{2} U \\
& -\frac{1}{M h}\left(\beta_{0}+\beta_{1} \widehat{z}^{N}-\alpha_{0}-\alpha_{1} \widehat{z}^{N}\right) \widehat{z}^{(1-M)} k \frac{d W}{d \widehat{z}} \\
& -\frac{1}{M h} \widehat{z}^{(1-M)} \alpha_{1} N \widehat{z}^{(N-1)} k W=0
\end{aligned}
$$

$$
\begin{aligned}
& \left(\frac{1}{M h}\right)^{2}\left(\beta_{0}+\beta_{1} \widehat{z}^{N}\right)\left[\widehat{z}^{2(1-M)} \frac{d^{2} W}{d \widehat{z}^{2}}+(1-M) \widehat{z}^{(1-2 M)} \frac{d W}{d \widehat{z}}\right] \\
& \quad+\left(\frac{1}{M h}\right)^{2} \widehat{z}^{2(1-M)} \beta_{1} N \widehat{z}^{(N-1)} \frac{d W}{d \widehat{z}} \\
& +\left[\left(\gamma_{0}+\gamma_{1} \widehat{z}^{N}\right) c^{2}-\left(\alpha_{0}+\alpha_{1} \widehat{z}^{N}\right)\right] k^{2} W \\
& \quad+\frac{1}{M h}\left(\beta_{0}+\beta_{1} \widehat{z}^{N}-\alpha_{0}-\alpha_{1} \widehat{z}^{N}\right) \widehat{z}^{(1-M)} k \frac{d U}{d \widehat{z}} \\
& \quad+\frac{1}{M h} \widehat{z}^{(1-M)}\left(\beta_{1}-2 \alpha_{1}\right) N \widehat{z}^{(N-1)} k U=0 .
\end{aligned}
$$

The solution for (8) is also assumed to be

$$
U=\sum_{n=0}^{\infty} r_{n} \widehat{z}^{n}, \quad W=\sum_{n=0}^{\infty} s_{n} \widehat{z}^{n}
$$

By substituting (15) into (14) and multiplying $\widehat{z}^{2 M-2}$ on both sides, we obtain

$$
\begin{aligned}
& \left(\frac{1}{M h}\right)^{2}\left(\alpha_{0}+\alpha_{1} \widehat{z}^{N}\right) \sum_{n=1}^{\infty}\left[n(n-M) r_{n} \widehat{z}^{n-2}\right] \\
& +\left(\frac{1}{M h}\right)^{2} \alpha_{1} N \widehat{z}^{N-1} \sum_{n=1}^{\infty} n r_{n} \hat{z}^{n-1} \\
& +\left[\left(\gamma_{0}+\gamma_{1} \widehat{z}^{N}\right) c^{2}-\left(\beta_{0}+\beta_{1} \widehat{z}^{N}\right)\right] k^{2} \widehat{z}^{2(M-1)} \sum_{n=0}^{\infty} r_{n} \widehat{z}^{n} \\
& -\frac{1}{M h} \alpha_{1} N \widehat{z}^{N+M-2} k \sum_{n=0}^{\infty} s_{n} \widehat{z}^{n} \\
& -\frac{1}{M h}\left(\beta_{0}+\beta_{1} \widehat{z}^{N}-\alpha_{0}-\alpha_{1} \widehat{z}^{N}\right) \widehat{z}^{M-1} k \sum_{n=1}^{\infty} n s_{n} \widehat{z}^{n-1}=0 \\
& \left(\frac{1}{M h}\right)^{2}\left(\beta_{0}+\beta_{1} \widehat{z}^{N}\right) \sum_{n=1}^{\infty}\left[n(n-M) s_{n} \widehat{z}^{n-2}\right] \\
& +\frac{1}{M h}\left(\beta_{1}-2 \alpha_{1}\right) N \widehat{z}^{N+M-2} k \sum_{n=0}^{\infty} r_{n} \widehat{z}^{n} \\
& +\left(\frac{1}{M h}\right)^{2} \beta_{1} N \widehat{z}^{N-1} \sum_{n=1}^{\infty} n s_{n} \widehat{z}^{n-1} \\
& +\left[\left(\gamma_{0}+\gamma_{1} \widehat{z}^{N}\right) c^{2}-\left(\alpha_{0}+\alpha_{1} \widehat{z}^{N}\right)\right] k^{2} \widehat{z}^{2(M-1)} \sum_{n=0}^{\infty} s_{n} \widehat{z}^{n} \\
& +\frac{1}{M h}\left(\beta_{0}+\beta_{1} \widehat{z}^{N}-\alpha_{0}-\alpha_{1} \widehat{z}^{N}\right) \widehat{z}^{M-1} k \sum_{n=1}^{\infty} n r_{n} \widehat{z}^{n-1}=0 .
\end{aligned}
$$

In (16), the lowest order of the power of $\widehat{z}$ is zero. The values of $r_{0}$ and $s_{0}$ do not influence the coefficients of $\widehat{z}^{0}$ in (16). Therefore, they are considered as undetermined constants of 
the solutions. By equating the coefficient of $\widehat{z}^{j},(0 \leq j \leq$ $M-2)$ in (16), we obtain

$$
r_{n}=0, \quad s_{n}=0, \quad(0<n<M),
$$

and $r_{M}$ and $s_{M}$ are also undetermined constants.

Furthermore, by equating the coefficients of $\widehat{z}^{j},(j \geq M-$ 1 ) in (16), a series of recursive equations are obtained as

$$
\begin{aligned}
& \left(\frac{1}{M h}\right)^{2}\left[\alpha_{0}(j+2)(j+2-M) r_{j+2}\right. \\
& \left.\quad+\alpha_{1}(j+2-N)(j+2-M) r_{j-N+2}\right] \\
& \quad-\frac{k}{M h}\left(\beta_{1}-\alpha_{1}\right)(j-M-N+2) s_{j-M-N+2} \\
& \quad-\frac{k}{M h}\left(\beta_{0}-\alpha_{0}\right)(j-M+2) s_{j-M+2} \\
& +\left(\gamma_{1} c^{2}-\beta_{1}\right) k^{2} r_{j-2 M-N+2}-\frac{k}{M h} \alpha_{1} N s_{j-M-N+2} \\
& +\left(\gamma_{0} c^{2}-\beta_{0}\right) k^{2} r_{j-2 M+2}=0 \\
& \left(\frac{1}{M h}\right)^{2}\left[\beta_{0}(j+2)(j-M+2) s_{j+2}\right. \\
& \left.\quad+\beta_{1}(j+2-N)(j-M+2) s_{j-N+2}\right] \\
& \quad+\frac{k}{M h}\left(\beta_{0}-\alpha_{0}\right)(j-M+2) r_{j-M+2} \\
& +\frac{k}{M h}\left(\beta_{1}-\alpha_{1}\right)(j-M-N+2) r_{j-M-N+2} \\
& +\frac{k}{M h} N\left(\beta_{1}-2 \alpha_{1}\right) r_{j-M-N+2} \\
& +\left(\gamma_{1} c^{2}-\alpha_{1}\right) k^{2} s_{j-2 M-N+2}+\left(\gamma_{0} c^{2}-\alpha_{0}\right) k^{2} s_{j-2 M+2}=0
\end{aligned}
$$

Therefore, the solution for (8) may be rewritten as

$$
U=r_{0}+\sum_{n=M}^{\infty} r_{n} \widehat{z}^{n}, \quad W=s_{0}+\sum_{n=M}^{\infty} s_{n} \widehat{z}^{n}
$$

where $r_{0}, r_{M}, s_{0}$, and $s_{M}$ are the independent unknown coefficients.

To simplify these independent unknown coefficients, the following matrix is introduced:

$$
\left(\begin{array}{llll}
r_{0 j} & r_{M j} & s_{0 j} & s_{M j}
\end{array}\right)=I,
$$

where $j=1 \sim 4$ and $I$ is a $4 \times 4$ unity matrix. Thus, (19) may be rewritten as

$$
\begin{gathered}
U=\sum_{j=1}^{4} C_{j}\left(r_{0 j}+r_{M j} \widehat{z}^{M}+\sum_{n=M+1}^{\infty} r_{n j} \widehat{z}^{n}\right), \\
W=\sum_{j=1}^{4} C_{j}\left(s_{0 j}+s_{M j} \widehat{z}^{M}+\sum_{n=M+1}^{\infty} s_{n j} \widehat{z}^{n}\right),
\end{gathered}
$$

where the constants $C_{j}(j=1 \sim 4)$ are to be determined. For $n=0$ and $n=M, r_{n j}$ and $s_{n j}$ are defined by (20). For the other values of $n, r_{n j}$ and $s_{n j}$ may be determined by solving (18), whereas $r_{n}$ and $s_{n}$ are replaced by $r_{n j}$ and $s_{n j}$ in these equations.

From (1), (3), and (21), we obtain the following stress components:

$$
\begin{aligned}
& \sigma_{z z}=i\left[\left(c_{11}-2 c_{44}\right) k U+\frac{c_{11}}{M h} \widehat{z}^{(1-M)} \frac{d W}{d \widehat{z}}\right] \exp [i k(x-c t)] \\
& \sigma_{x z}=c_{44}\left(\frac{1}{M h} \widehat{z}^{(1-M)} \frac{d U}{d \widehat{z}}-k W\right) \exp [i k(x-c t)] .
\end{aligned}
$$

By substituting (21) into the boundary conditions, we then obtain a set of homogeneous linear algebraic equations for the undetermined constants $C_{i}(i=1 \sim 4)$. The sufficient and necessary condition for the existence of a nontrivial solution is that the determinant of the coefficient matrix has to vanish. The dispersion equation is

$$
\left|Q_{i j}\right|=0
$$

where $i=1 \sim 4, j=1 \sim 4$, and

$$
\begin{aligned}
Q_{1 j} & =k\left(c_{110}-2 c_{440}\right) r_{0 j}+\frac{c_{110}}{h} s_{M j}, \quad Q_{2 j}=\frac{r_{M j}}{h}+k s_{0 j}, \\
Q_{3 j} & =\left(c_{11 h}-2 c_{44 h}\right) k \sum_{n=0}^{\infty} r_{n j}+\frac{c_{11 h}}{M h} \sum_{n=0}^{\infty} n s_{n j}, \\
Q_{4 j} & =\frac{1}{M h} \sum_{n=0}^{\infty} n r_{n j}-k \sum_{n=0}^{\infty} s_{n j} .
\end{aligned}
$$

\section{Numerical Example and Discussion}

Based on the dispersion relation in (23), numerical examples are given to illustrate the propagation behavior of Lamb waves in an FGM plate. In the numerical analysis, $\mathrm{Cr}$ and ceramic are chosen as materials I and II, respectively. Their material parameters are [18] as follows:

Cr:

$$
\begin{aligned}
& \rho^{(1)}=7190 \mathrm{~kg} / \mathrm{m}^{3}, \quad c_{44}^{(1)}=102.5 \mathrm{GPa}, \\
& c_{11}^{(1)}=279.2 \mathrm{GPa}
\end{aligned}
$$

Ceramics:

$$
\begin{aligned}
& \rho^{(2)}=3900 \mathrm{~kg} / \mathrm{m}^{3}, \quad c_{44}^{(2)}=118.11 \mathrm{GPa}, \\
& c_{11}^{(2)}=374.9 \mathrm{GPa} .
\end{aligned}
$$

Normally, the demission of elastic coefficients is different to that of density, and the longitudinal wave velocity and the shear wave velocity are dependent on the ratio of the elastic coefficients to the density. Therefore, in this study, we 


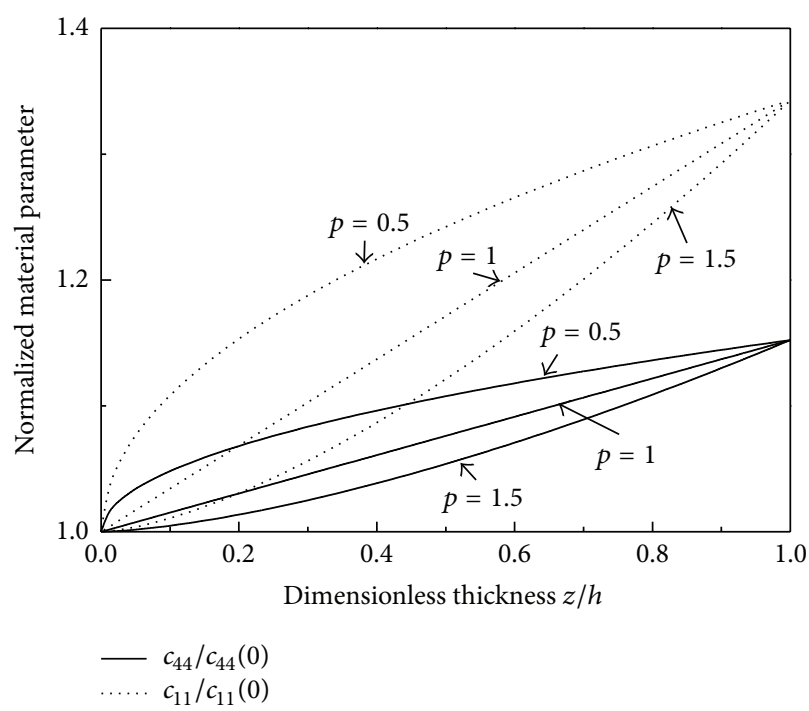

(a)

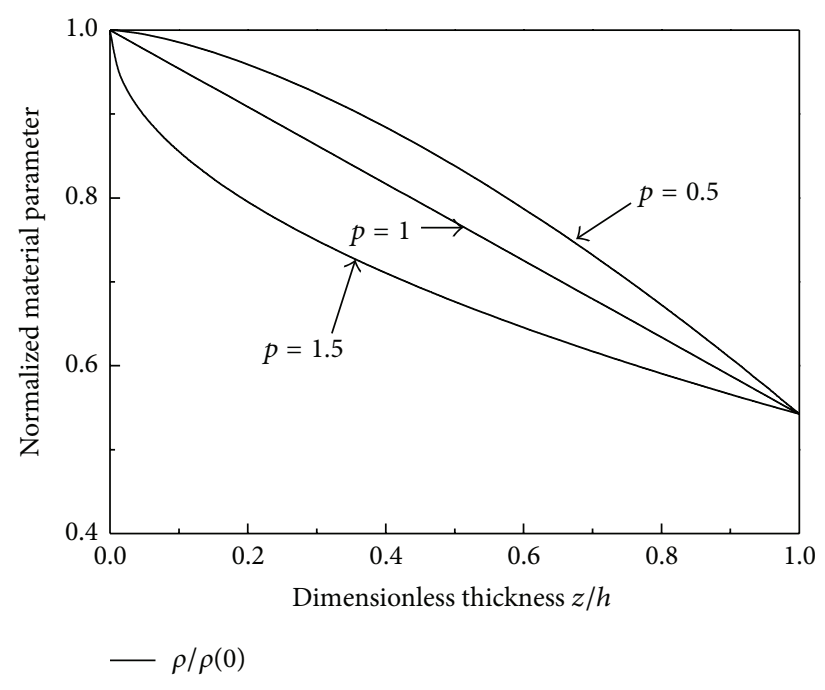

(b)

FIGURE 2: Profiles of material parameters: (a) normalized elastic parameter $c_{11} / c_{11}(0)$ and $c_{44} / c_{44}(0)$ and (b) normalized density $\rho / \rho(0)$.

presented these in a nondimensional way. The profiles of the nondimensional material properties that vary with thickness are shown in Figure 2.

The dispersion curves of the first four modes are plotted in Figure 3, where the dimensionless wave number $k h$ and phase velocity $c$ are used as the abscissa and ordinate, respectively. As shown in Figure 3, in each mode, the phase velocity of Lamb waves in the FGM plate with $p=0.5$ is the largest, followed by that in the FGM plate with $p=1$. The phase velocity in the FGM plate with $p=1.5$ is the smallest. This phenomenon suggests that the phase velocity decreases when the gradient coefficient increases. For the convenience of nondestructive evaluation, the continuous relation between the phase velocity of Lamb waves in the FGM plate and the gradient coefficient is plotted in Figure 4. The dimensionless wave number $k h$ being $\pi$ and $2 \pi$ signifies that the thickness of the plate is equal to half of one wavelength and one wavelength, respectively. The influence of the gradient coefficient on the phase velocity is nonlinear. The result is in good agreement with that in [18], which discusses the discrete relation between the phase velocity and the gradient coefficient.

The dispersion properties are determined by the relationship between the phase velocity and the group velocity, which is defined as $c_{g}=d \omega / d k=c+d c / d k$. The physical meaning of the group velocity is the rate at which energy is transported. When the group velocity is greater than the phase velocity, this phenomenon is called anomalous dispersion. The opposite is called normal dispersion. When Lamb waves propagate in a homogenous plate, only anomalous dispersions occur in the first modes; normal dispersions exist in the second modes. When the Lamb waves propagate in an FGM plate, both anomalous and normal dispersions occur in the first and second modes. For example, in the first mode, the value of $d c / d k$ varies from positive to negative. Thus, a point at which

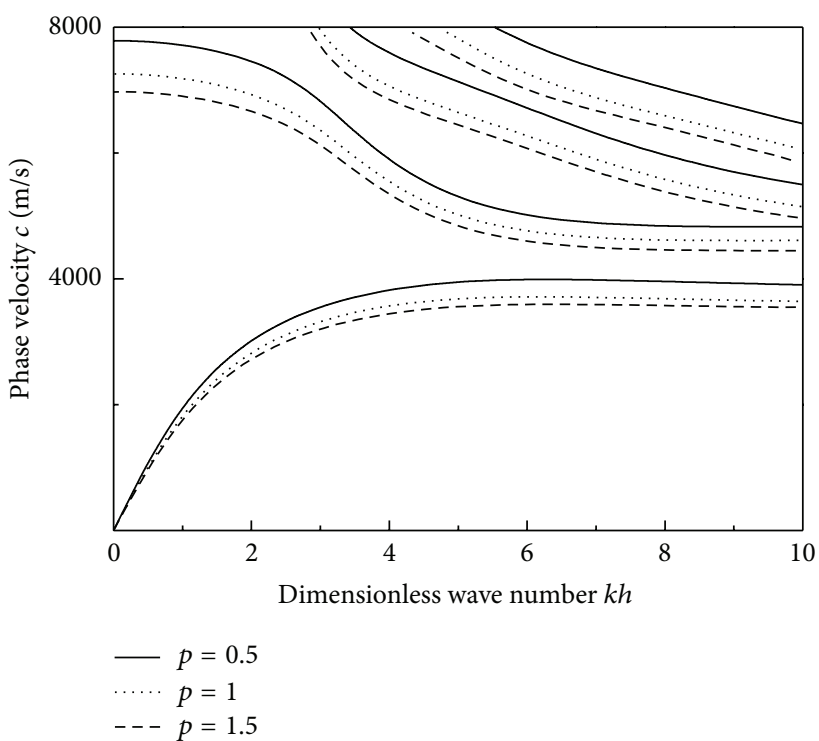

FIGURE 3: Dispersion curves of first four modes of Lamb waves in FGM plate.

$d c / d k$ is equal to zero exists. The phase velocity is implied to be equal to the group velocity at that point. We define the frequency of that point as the nondispersion frequency. The relation between gradient coefficient and the nondispersion frequencies of the first mode, $\bar{\omega}$, is plotted in Figure 5, where the products of nondispersion frequency and thickness $\bar{\omega} h$ are used as the ordinate. The nondispersion frequency is not monotonic and reaches minimum when the gradient coefficient approximately equals 1 . It implies that the nondispersion frequencies reach minimum when the variation rate of the material properties is constant. With wave number increases, 


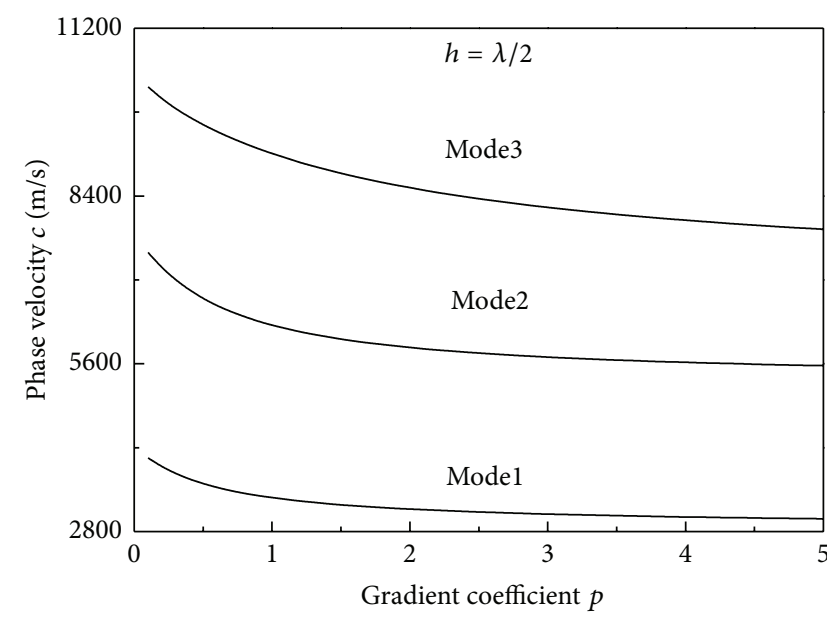

(a)

FIGURE 4: Phase velocity plotted as functions of gradient coefficient $p$ the plate equals a wavelength.

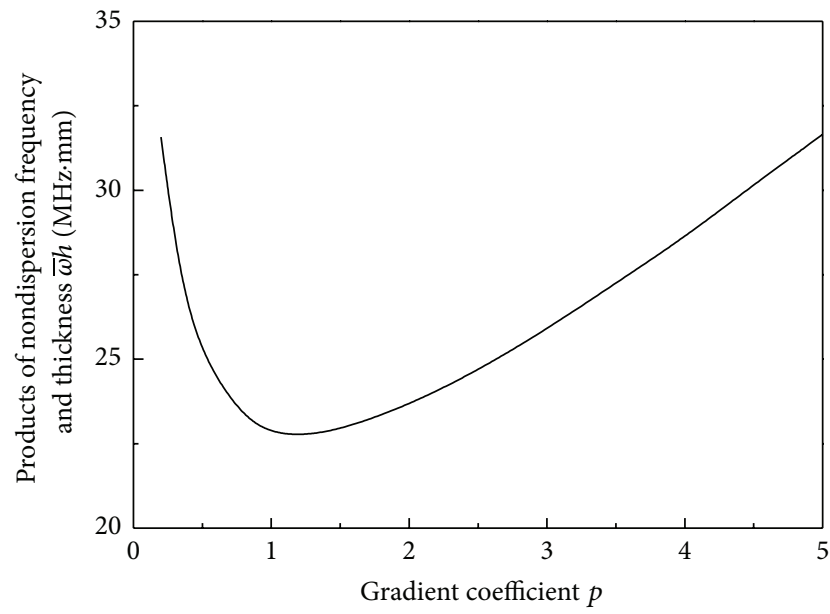

FIGURE 5: The relation between gradient coefficient and the nondispersion frequencies of Lamb waves in the first mode.

the earliest peak of dispersion curves of the first mode appears when the material properties vary along thickness linearly.

Many interesting aspects of mode cutoff frequency may be used for corrosion detection and thickness measurement in a variety of different structures. For Lamb waves, frequency values occur whenever standing longitudinal or shear waves are present across the thickness of the plate. We select a series of small dimensionless wave numbers $k h$ from 0.1 to 0.00001 and calculate the phase velocity. The limitation of the product of phase velocity and dimensionless wave number $k h$ at $k h \rightarrow$ 0 is the product of the cutoff frequency and thickness $\bar{\omega}_{n} h$.

Table 1 lists products of thickness and cutoff frequencies $\bar{\omega}_{n} h$ for the Lamb waves propagating in the homogenous plate and the FGM plate. Table 1 shows that the set of the cutoff frequencies could be considered to be a union of two series of approximate arithmetic progressions. In Table 1, the series

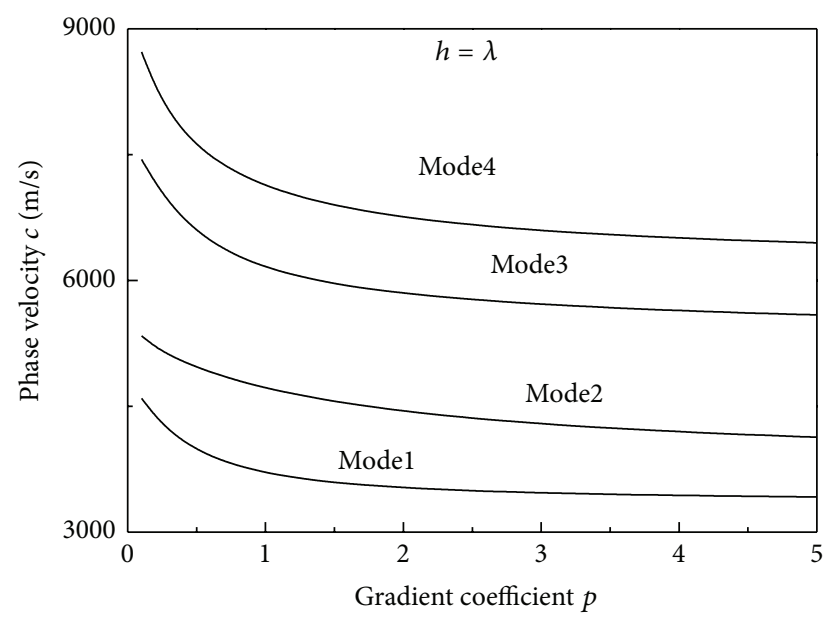

(b)

(a) Thickness of the plate equals half a wavelength. (b) Thickness of

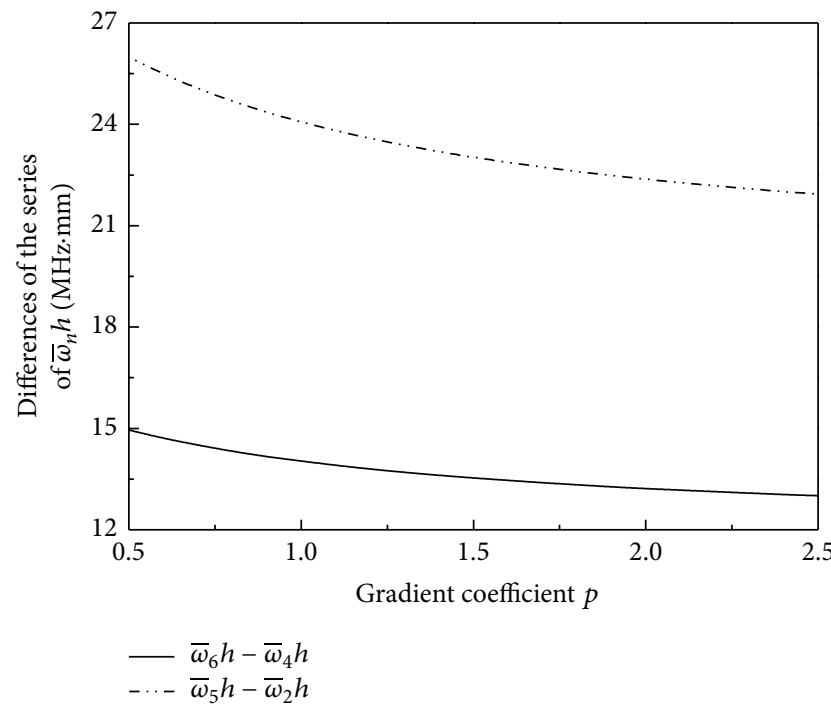

FIGURE 6: The relation between the differences of the series and the gradient coefficient.

of $\bar{\omega}_{n} h, n=1,3,4,6$, should be considered as one series of approximate arithmetic progressions. The differences approximately are $14.9,14,13.5$, and $13 \mathrm{MHz} \cdot \mathrm{mm}$ when the gradient coefficient is $0.5,1,1.5$, and 2.5 , respectively. While the series of $0, \bar{\omega}_{n} h, n=2,5$, should be considered as another series of approximate arithmetic progression, the differences approximately are $26,24,23$, and $22 \mathrm{MHz} \cdot \mathrm{mm}$ when the gradient coefficient is $0.5,1,1.5$, and 2.5 , respectively. Let $\bar{\omega}_{6} h-$ $\bar{\omega}_{4} h$ and $\bar{\omega}_{5} h-\bar{\omega}_{2} h$ represent the differences of these series, respectively. We plot the relation between the differences of the series and the gradient parameter in Figure 6. The differences decrease as the gradient coefficient increases. It suggests that the gradient coefficient should be evaluated from the cutoff frequencies. 
TABLE 1: Products of thickness and cutoff frequencies of the Lamb waves propagating in the homogenous plate and the FGM plate $\bar{\omega}_{n} h$ (MHz.mm).

\begin{tabular}{|c|c|c|c|c|c|c|}
\hline$n$ & $\mathrm{Cr}$ & Ceramics & $\operatorname{FGM}(p=0.5)$ & $\operatorname{FGM}(p=1)$ & $\operatorname{FGM}(p=1.5)$ & FGM $(p=2.5)$ \\
\hline 1 & 11.862 & 17.289 & 14.931 & 14.135 & 13.708 & 13.233 \\
\hline 2 & 19.577 & 30.802 & 26.013 & 24.243 & 23.267 & 22.195 \\
\hline 3 & 23.723 & 34.577 & 29.855 & 28.287 & 27.173 & 26.173 \\
\hline 4 & 35.585 & 51.866 & 44.832 & 42.132 & 40.675 & 39.142 \\
\hline 5 & 39.154 & 61.603 & 51.988 & 48.228 & 46.202 & 44.071 \\
\hline 6 & 47.447 & 69.155 & 59.790 & 56.154 & 54.192 & 52.125 \\
\hline
\end{tabular}

\section{Conclusion}

Variable substitution and the power series technique are used to solve Lamb waves' propagation problems in a functionally graded composite with nonintegral power function volume fractions. The continuous relation between the phase velocity and the gradient coefficients is obtained. The numerical results show that the phase velocity decreases as the gradient coefficient increases, nondispersion frequency exists in the first mode, and the set of cutoff frequencies is related to the gradient coefficient. Based on these results, three potential methods may be employed for nondestructive evaluation based on Lamb waves. These methods include phase velocity, nondispersion frequency, and cutoff frequency. The present theoretical study not only provides a method for solving the wave propagation problems in an FGM used in the thermal protection systems but also serves as guide for ultrasonic nondestructive evaluation.

\section{Conflict of Interests}

The authors declare that there is no conflict of interests regarding the publication of this paper.

\section{Acknowledgments}

The authors gratefully acknowledge the support by the National Natural Science Foundation of China (Nos. 10902079 and 11302166) and Special Funds for the Development of Characteristic Key Disciplines in the Local University by the Central Financial Support.

\section{References}

[1] M. Koizumi, "The concept of FGM," in Ceramic Transaction, Functionally Gradient Materials, vol. 34, pp. 3-10, 1993.

[2] C. F. Lü, C. W. Lim, and W. Q. Chen, "Size-dependent elastic behavior of FGM ultra-thin films based on generalized refined theory," International Journal of Solids and Structures, vol. 46, no. 5, pp. 1176-1185, 2009.

[3] A. J. Markworth and J. H. Saunders, "A model of structure optimization for a functionally graded material," Materials Letters, vol. 22, no. 1-2, pp. 103-107, 1995.

[4] Y. Fukui, K. Takashima, and C. B. Ponton, "Measurement of Young's modulus and internal friction of an in situ Al-Al3Ni functionally gradient material," Journal of Materials Science, vol. 29, no. 9, pp. 2281-2288, 1994.
[5] F. Erdogan, "Fracture mechanics of functionally graded materials," Composites Engineering, vol. 5, no. 7, pp. 753-770, 1995.

[6] J. N. Reddy and C. D. Chin, "Thermomechanical analysis of functionally graded cylinders and plates," Journal of Thermal Stresses, vol. 21, no. 6, pp. 593-626, 1998.

[7] J. Zhu, G. R. Ye, Y. Q. Xiang, and W. Q. Chen, "Recursive formulae for wave propagation analysis of FGM elastic plates via reverberation-ray matrix method," Composite Structures, vol. 93, no. 2, pp. 259-270, 2011.

[8] G. R. Liu, X. Han, and K. Y. Lam, "Material characterization of FGM plates using elastic waves and an inverse procedure," Journal of Composite Materials, vol. 35, no. 11, pp. 954-971, 2001.

[9] G. R. Liu, J. Tani, and T. Ohyoshi, "Lamb waves in a functionally gradient material plate and its transient response (part 1: theory)," Transactions of the Japan Society of Mechanical Engineers, vol. 57, no. 535, pp. 603-608, 1991.

[10] X. Han, G. R. Liu, K. Y. Lam, and T. Ohyoshi, "Quadratic layer element for analyzing stress waves in FGMS and its application in material characterization," Journal of Sound and Vibration, vol. 236, no. 2, pp. 307-321, 2000.

[11] X. Han, G. R. Liu, Z. C. Xi, and K. Y. Lam, "Characteristics of waves in a functionally graded cylinder," International Journal for Numerical Methods in Engineering, vol. 53, no. 3, pp. 653676, 2002.

[12] G. R. Liu, X. Han, and K. Y. Lam, "Stress waves in functionally gradient materials and its use for material characterization," Composites B: Engineering, vol. 30, no. 4, pp. 383-394, 1999.

[13] X. Y. Li, Z. K. Wang, and S. H. Huang, "Love waves in functionally graded piezoelectric materials," International Journal of Solids and Structures, vol. 41, no. 26, pp. 7309-7328, 2004.

[14] V. Vlasie and M. Rousseau, "Guided modes in a plane elastic layer with gradually continuous acoustic properties," NDT and E International, vol. 37, no. 8, pp. 633-644, 2004.

[15] X. Cao, F. Jin, and Z. Wang, "Theoretical investigation on horizontally shear waves in a functionally gradient piezoelectric material plate," Advanced Materials Research, vol. 33-37, pp. 707-712, 2008.

[16] J. Liu and Z. K. Wang, "Study on the propagation of Rayleigh waves in a graded half space," Chinese Journal of Applied Mechanics, vol. 21, pp. 106-109, 2008.

[17] B. Wu, J. Yu, and C. He, "Wave propagation in nonhomogeneous magneto-electro-elastic plates," Journal of Sound and Vibration, vol. 317, no. 1-2, pp. 250-264, 2008.

[18] X. Cao, F. Jin, and I. Jeon, "Calculation of propagation properties of Lamb waves in a functionally graded material (FGM) plate by power series technique," NDT \& E International, vol. 44, no. 1, pp. 84-92, 2011. 

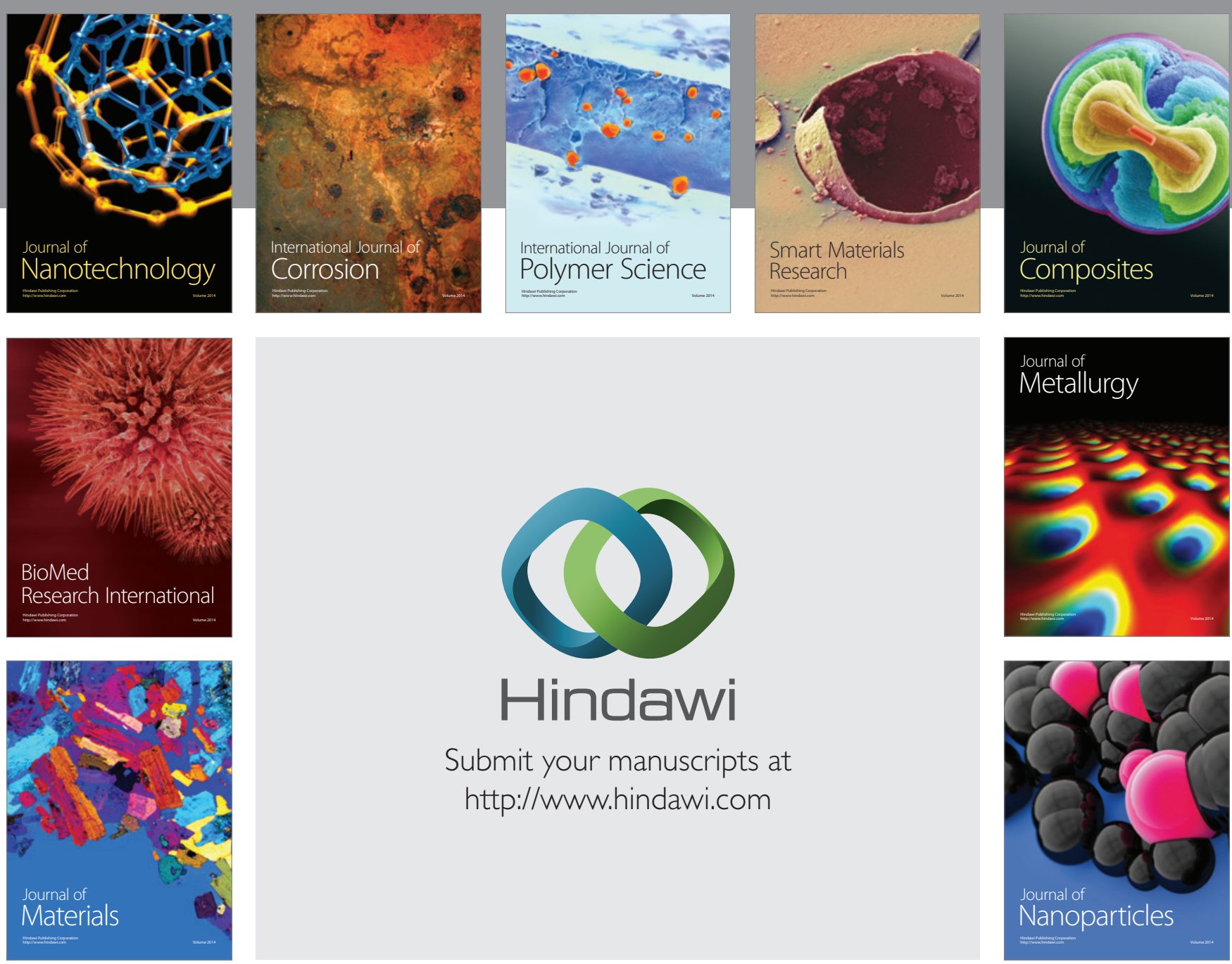

Submit your manuscripts at http://www.hindawi.com
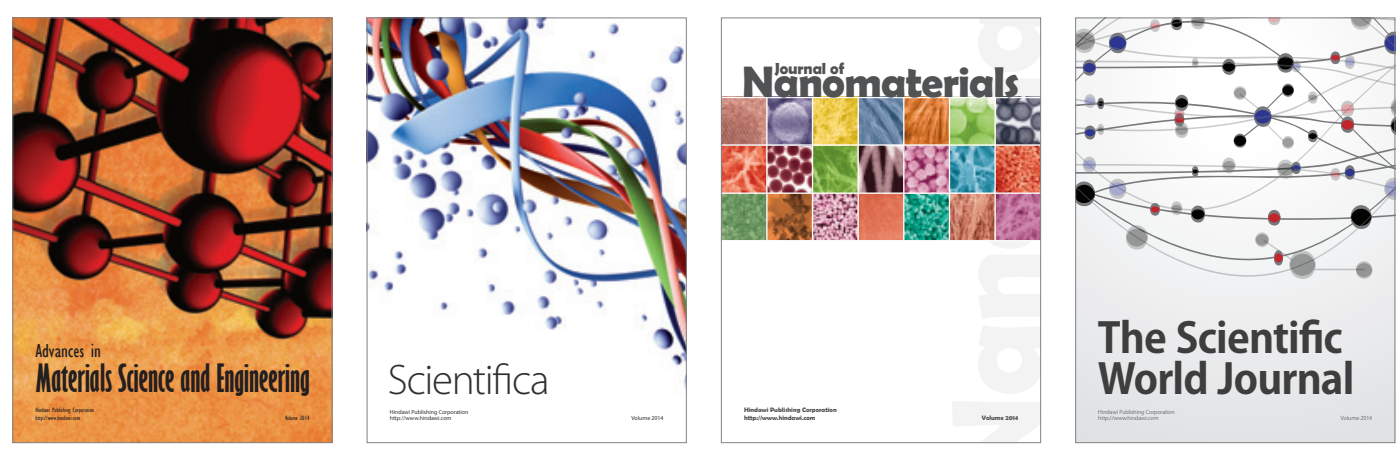

\section{The Scientific World Journal}
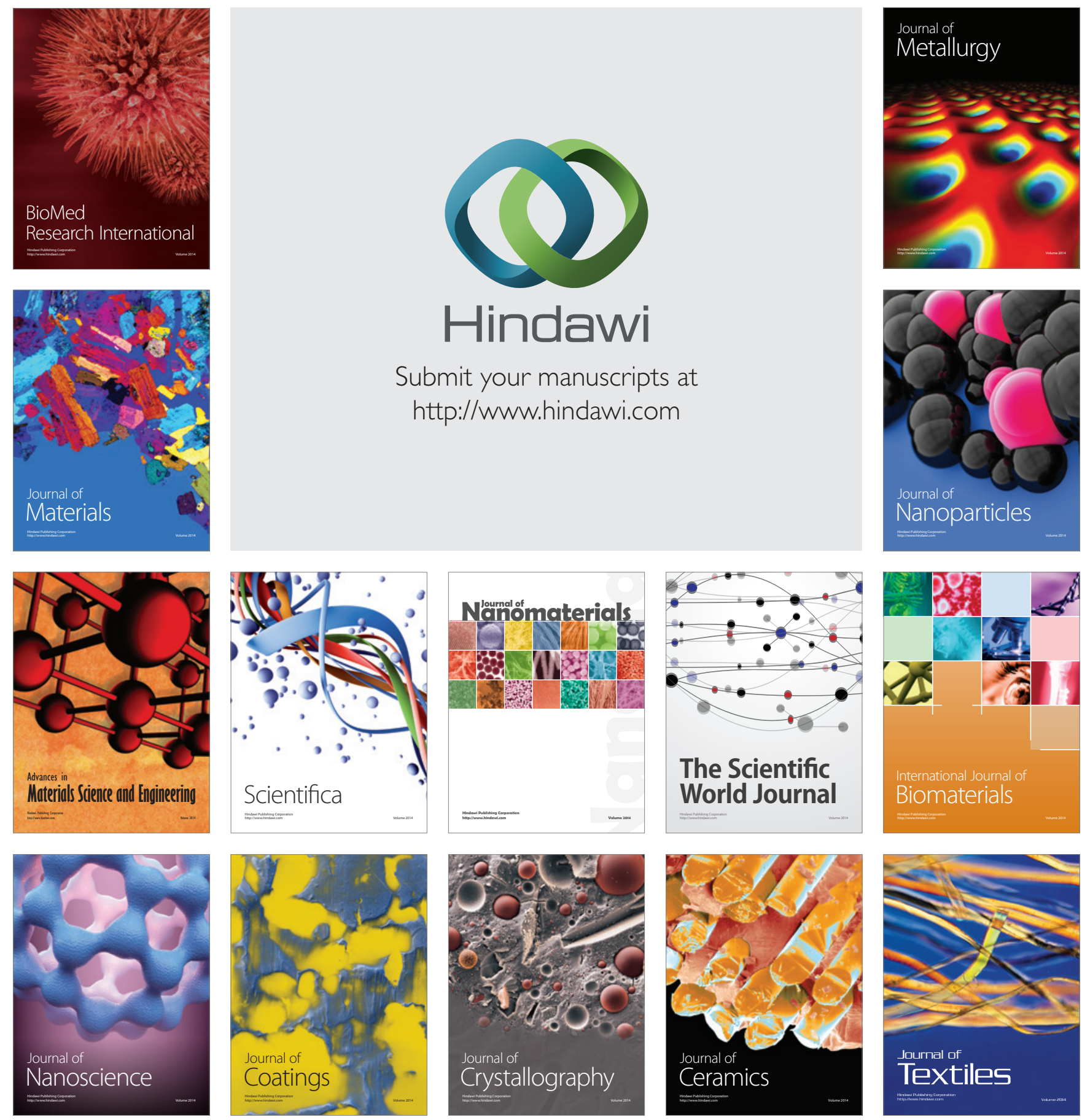